\title{
Adquisición de datos de enfriamiento con termopar tipo $k$ para probetas de acero inoxidable en la plataforma Arduino
}

\section{Data acquisition of cooling using k-type thermocouple for stainless steel specimens on the Arduino platform}

NÚÑEZ-ARIZA, Benjamín†, NÚÑEZ-HERNÁNDEZ, Lourdes Nohemí, YÁÑEZ-VARGAS, Juan Israel y KANTUN-UICAB, María Cristina*

Universidad Politécnica de Juventino Rosas, Maestría en Ingeniería, Fabricación y Materiales Avanzados.

ID $1^{\text {st }}$ Author: Benjamín, Núñez-Ariza / ORC ID: 0000-0002-6941-1903, CVU CONACYT ID: 1017742

ID $1^{\text {st }}$ Coauthor: Lourdes Nohemí, Núñez-Hernández / ORC ID: 0000-0002-3684-7700

ID $2^{\text {nd }}$ Coauthor: Juan Israel, Yáñez-Vargas / ORC ID: 0000-0001-5749-8442, CVU CONACYT ID: 295711

ID $3^{\text {rd }}$ Coauthor: María Cristina, Kantun-Uicab / ORC ID: 0000-0003-1588-5414, CVU CONACYT ID: 162342

DOI: $10.35429 / J E A .2020 .23 .7 .19 .23$

Recibido: 10 de Abril, 2020; Aceptado: 30 de Junio, 2020

\begin{abstract}
Resumen
En el presente trabajo se realizó un estudio experimental en la adquisición de datos, de las temperaturas dentro de un horno marca Nobertherm P300 de resistencias, utilizando un termopar flexible Tipo-K usando la plataforma Arduino, y el procesamiento de datos es el software Matlab. Se utilizaron probetas de acero inoxidable (1/2" de diámetro por 2" de largo) como material de prueba. Los termopares fueron instalados dentro de las probetas a una profundidad de 1", después se colocaron dentro de un horno precalentado a la temperatura de $900{ }^{\circ} \mathrm{C}$. El proceso de enfriamiento se realizó en un recipiente con agua a temperatura ambiente por 20 segundos. Los datos de enfriamiento se monitorearon con el Arduino Mega 2560 a través de módulos de lectura de termopar MAX 6675.El resultado obtenido durante el periodo de enfriamiento estudiado, es de seis lecturas por segundo. Esto podría ser una precisión comparable con equipos comerciales. Además, el procesamiento de datos se hizo en la plataforma de Matlab® dentro de una aplicación, donde se mostrarán los gráficos de las distintas pruebas.
\end{abstract}

Adquisición de Datos, Termopar tipo K, Arduino Mega 2560

\begin{abstract}
In the present work, was made an experimental study in the acquisition of data of the temperature in a furnace brand Nobertherm P300 of resistance, using a thermocouple flexible K-Type using the platform, Arduino, and the processing of data is in the software matlab. It was used stainless steel cylindrical $(1 / 2$ " diameter per 2" long) specimens as test material. The thermocouples were installed inside the probes in a depth of 1 inch, then were placed in the pre-heated furnace to $900{ }^{\circ} \mathrm{C}$. The cooling process was carried out with water at room temperature for 20 seconds. Cooling data were monitored employing an Arduino Mega 2560 connected to thermocouple reading modules MAX 6675. The results obtained were six readings per second in the cooling period studied. This could be a comparable precision to commercial equipment. Also, the data processing was done on the Matlab® platform within an application, where the graphics generated from the different test will be shown.
\end{abstract}

Acquisition of data, thermocouple K-Type and Arduino Mega 2560

Citación: NÚÑEZ-ARIZA, Benjamín, NÚÑEZ-HERNÁNDEZ, Lourdes Nohemí, YÁÑEZ-VARGAS, Juan Israel y KANTUN-UICAB, María Cristina. Adquisición de datos de enfriamiento con termopar tipo $\mathrm{k}$ para probetas de acero inoxidable en la plataforma Arduino. Revista de Aplicaciones de la Ingeniería. 2020. 7-23:19-23.

\footnotetext{
* Correspondencia del Autor (Correo Electrónico: mkantun_ptc@upjr.edu.mx)

$\dagger$ Investigador contribuyendo como primer autor.
} 


\section{Introducción}

El procesamiento de información en las industrias es pieza fundamental, debido a que por medio de estos datos las empresas toman decisiones cruciales. Por otro lado, el manejar y mostrar datos de máquinas a través de una interface gráfica, es una tarea difícil de acoplar a las tecnologías tradicionales, ya que se les deben hacer modificaciones considerables pero necesarias para las exigentes políticas actuales del mercado.

De la página oficial de NATIONAL INSTRUMENTS nos dice que:

La adquisición de datos (DAQ) es el proceso de medir con una PC un fenómeno eléctrico o físico como voltaje, corriente, temperatura, presión o sonido. Un sistema DAQ consiste de sensores, hardware de medidas DAQ y una PC (Figura 1) con software programable. Comparados con los sistemas de medidas tradicionales, los sistemas DAQ basados en PC aprovechan la potencia del procesamiento, la productividad, la visualización y las habilidades de conectividad de las PCs estándares en la industria proporcionando una solución de medidas más potente, flexible y rentable. (C)2020 National Instruments Corporation

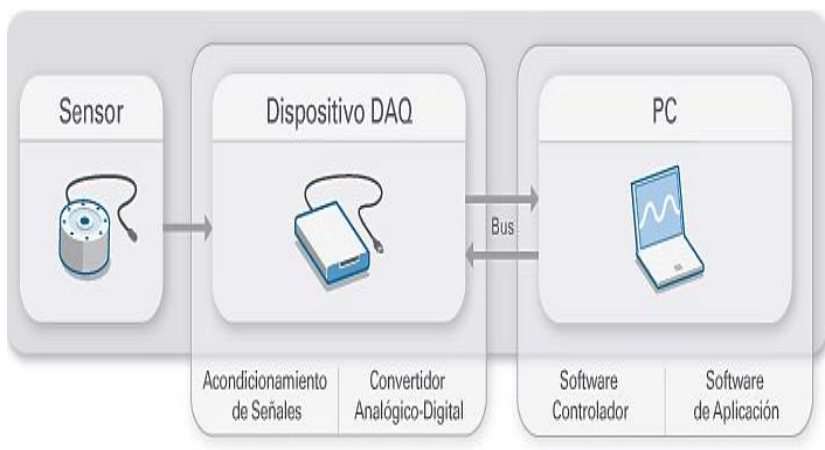

Figura 1 Estructura de un sistema de adquisición de datos Fuente: imagen obtenida de la página oficial de NATIONAL INSTRUMENTS

Por lo tanto, el propósito de los equipos de adquisición de datos es la reducción de rechazos, ya que la manufactura actual, exige que el total de un determinado lote de piezas o un proceso se analice al cien por ciento.

Del proceso de manufactura que actualmente nos exige que se obtenga el registro de cada variable presente y por medio de esta, se podrá determinar acciones sobre el material e incluso permitir garantizar que el proceso se cumplió dentro de los parámetros establecidos.
El surgimiento de las tecnologías y el diseño de nuevos sensores apoyan de manera conjunta para la obtención de estos datos. A través del presente documento se mostrará una alternativa acerca de estos dispositivos, en vista de que marcas distinguidas y comerciales no son tan accesibles para empresas en desarrollo.

\section{Metodología}

El equipo se desarrolló en tres etapas: la primera consiste en la instalación de los equipos que servirán para la recolección de información, la segunda es la construcción del equipo y las probetas que van a ser sometidas al calor y por último la etapa de procesamiento de los datos que tendrá una interfaz gráfica en Matlab ${ }^{\circledR}$ en la aplicación guide.

El uso de la plataforma Arduino, tiene como objetivo mostrar la eficiencia en velocidad de recolección de datos a bajo costo, ya que esta plataforma es totalmente gratuita. Por lo tanto, la construcción del equipo se desarrolló sobre el modelo Mega 2560 de Arduino, la razón por la cual se elige esta placa es por contar con suficientes puertos digitales para el procesamiento de información que será recibida por los cinco módulos de comunicación Arduino con termopar el Max 6675 y posteriormente envidada al módulo de lectura de tarjetas SD dónde serán guardados los datos. Esta placa por ergonomía incluye los puertos designados para el lector de tarjetas SD, así como para la pantalla LCD. El objetivo de esta última nos permitió interactuar de forma visual con la plataforma y observar de manera instantánea si existe un problema con un módulo o termopar y actuar de inmediato y corregir cualquier anomalía. Por último, todo el equipo fue montado sobre dos hojas de acrílico de $6 \mathrm{~mm}$, con la finalidad de proteger el equipo de impacto y del polvo (Figura 2).

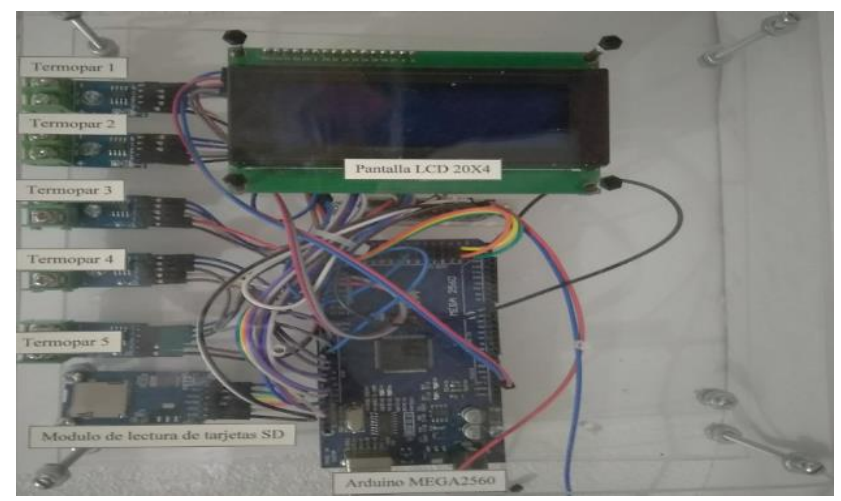

Figura 2 Equipo totalmente instalado y etiquetado Fuente: imagen del Autor Ing. Benjamín Núñez Ariza

NÚÑEZ-ARIZA, Benjamín, NÚÑEZ-HERNÁNDEZ, Lourdes Nohemí, YÁÑEZ-VARGAS, Juan Israel y KANTUN-UICAB, María Cristina. Adquisición de datos de enfriamiento con termopar tipo k para probetas de acero inoxidable en la plataforma Arduino. Revista de Aplicaciones de la Ingeniería. 2020 
Por otro lado, se fabricaron las probetas de prueba para llevar a cabo el experimento, estas probetas están bajo la norma de templabilidad aceros ASTM 205-02 la cual nos indica que dimensiones son las correctas para efectuar dicho experimento (Figura 3).

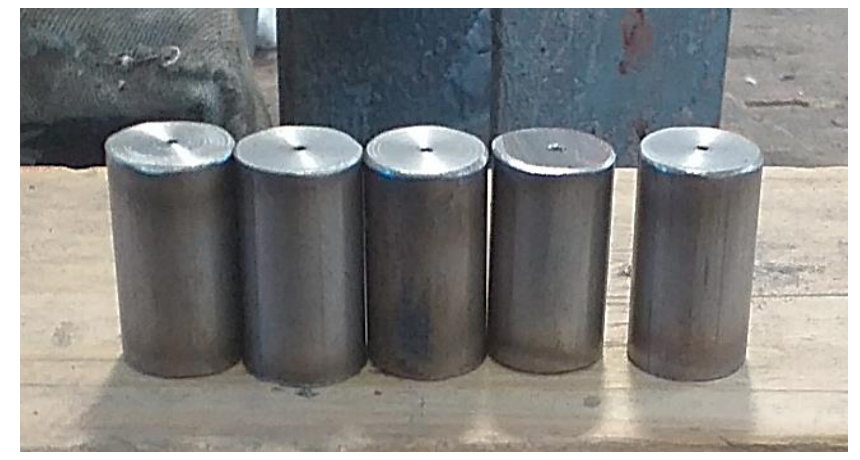

Figura 3 Probetas a utilizar durante los diversos experimentos en el cual es visible el barreno dónde fueron instalados los termopares

Fuente: imagen del Autor Ing. Benjamín Núñez Ariza

Para el caso particular de este trabajo, se utilizaron probetas de acero inoxidable con el objetivo de no generar residuo en cada prueba, pues de acuerdo con la siguiente definición se tomó esa decisión.

Los aceros inoxidables se seleccionan por su excelente resistencia a la corrosión. Todo verdadero acero inoxidable contiene un mínimo de aproximadamente $11 \%$ de $\mathrm{Cr}$, lo que permite una superficie delgada y protectora capa de óxido de cromo para formar cuando el acero está expuesto al oxígeno. El cromo es lo que hace que los aceros inoxidables sean inoxidables.

(Askeland y Wright, 2016, p. 485).

Ahora que se ha definido el motivo por el cual se empleó el acero inoxidable. Se continúa con la construcción de una estructura de material PTR en dónde se fijarán las probetas para ser introducidas en el horno y llevar a cabo los experimentos (Figura 4).

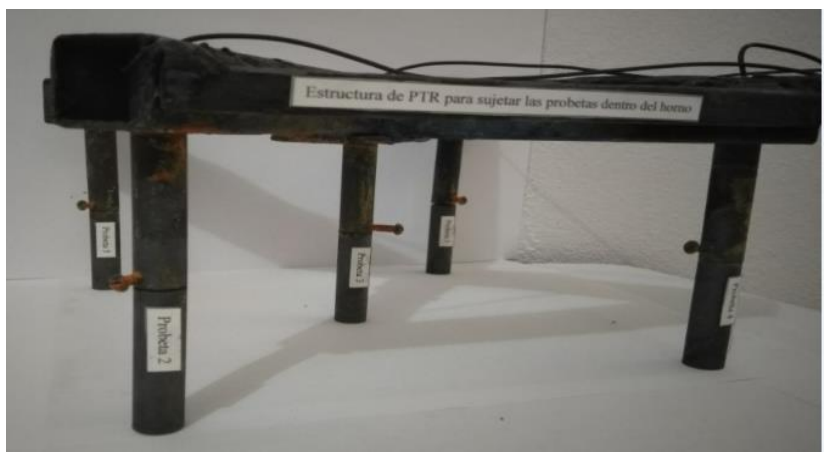

Figura 4 Equipo dónde fueron instaladas las probetas Fuente: imagen del Autor Ing. Benjamín Núñez Ariza

ECORFAN® Todos los derechos reservados
Para culminar esta etapa, se instaló un tanque de 60 litros con agua a una temperatura ambiente promedio de $22{ }^{\circ} \mathrm{C}$, en dónde fueron enfriadas súbitamente las probetas. Por último, la etapa del procesamiento de los datos se hizo en una aplicación diseñada en Matlab®, (Figura 5), la única modificación que se le efectuó a los datos que se encontraban en la memoria SD, es en relación con una conversión de tipo de archivo tex a uno dat para que puedan ser reconocidos por Matlab®.

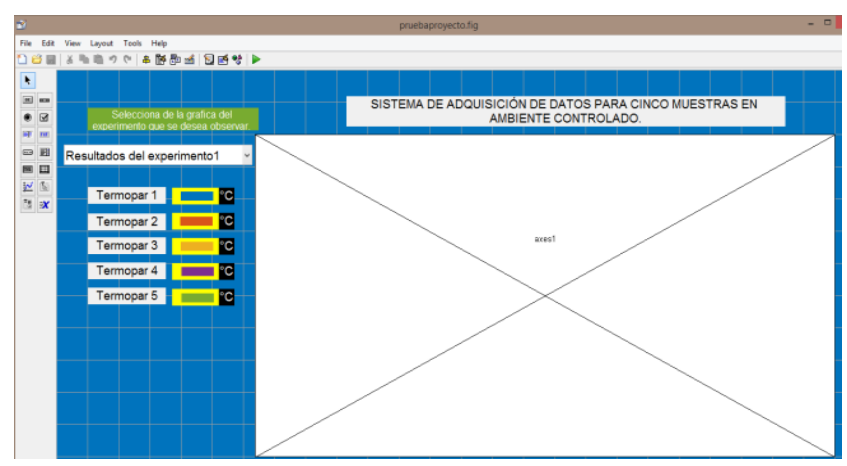

Gráfico 1 Pantalla de desarrollo para la aplicación del procesamiento de los datos

Fuente: elaboración propia

\section{Resultados}

Después de una serie de seis experimentos con un lapso promedio de una hora entre cada uno de ellos, se han recopilado los siguientes datos.

\begin{tabular}{|c|c|c|c|c|c|}
\hline seg & T1 & $\mathbf{T} 2$ & T3 & T4 & T5 \\
\hline \multirow{6}{*}{1} & 886 & 880.25 & 892.25 & 872.75 & 884.25 \\
\hline & 882.25 & 880.5 & 888.75 & 870 & 882 \\
\hline & 878 & 880.1 & 886.75 & 867.5 & 878.75 \\
\hline & 874.5 & 872.25 & 882.75 & 863.5 & 876 \\
\hline & 870.5 & 872 & 870.75 & 851 & 820 \\
\hline & 831.5 & 856 & 822.25 & 815.75 & 784.25 \\
\hline \multirow{6}{*}{2} & 744.5 & 735.5 & 722.25 & 716 & 688.5 \\
\hline & 632.5 & 688 & 592.75 & 592.75 & 582 \\
\hline & 536 & 564.25 & 499.5 & 479.75 & 483.25 \\
\hline & 453.5 & 464 & 417.5 & 401 & 412 \\
\hline & 392.75 & 384.35 & 346.75 & 338.75 & 356.25 \\
\hline & 344.5 & 332 & 299.25 & 283.25 & 313.5 \\
\hline \multirow{6}{*}{3} & 304.5 & 284.75 & 263.75 & 242.75 & 279.25 \\
\hline & 272.5 & 248.75 & 240.75 & 205.25 & 246.25 \\
\hline & 248 & 216 & 213.5 & 180.75 & 222.75 \\
\hline & 227 & 192 & 186.25 & 163.75 & 203.5 \\
\hline & 208 & 168.25 & 169 & 147 & 185.75 \\
\hline & 193.75 & 152 & 157.25 & 132.25 & 169.75 \\
\hline \multirow{6}{*}{4} & 181 & 144 & 147.25 & 120.75 & 157.5 \\
\hline & 168.25 & 128.75 & 139 & 111.75 & 146.75 \\
\hline & 161 & 120 & 132.25 & 102 & 136.5 \\
\hline & 148.5 & 112.5 & 123.75 & 93.75 & 122 \\
\hline & 136.75 & 104 & 118.5 & 88.25 & 107.25 \\
\hline & 129.5 & 92 & 118.25 & 83.5 & 101.75 \\
\hline
\end{tabular}

Tabla 1 Datos obtenidos del experimento uno Fuente: elaboración propia [MatLab] 
De acuerdo con la Tabla 1, en los primeros cuatro segundos se observa el enfriamiento súbito, dónde se obtuvieron un total de 24 lecturas por cada termopar, en ese lapso se asume que paso de una temperatura de $880{ }^{\circ} \mathrm{C}$ a una temperatura de $100{ }^{\circ} \mathrm{C}$ en promedio, ya que ambas temperaturas son relativas de acuerdo a los factores de posición dentro del horno y al enfriamiento súbito con el agua.

De los experimentos se puede percibir el cambio abrupto de las temperaturas de las probetas. Y una vez realizados una serie de experimentos, se determina que nuestro equipo, tiene la capacidad de recolectar información a una velocidad considerada con relación a los equipos comerciales. De tales experimentos se logró la obtención de seis lecturas por segundo como ya se expuso anteriormente. En el experimento 1 se obtuvo el Gráfico 2, en dónde se puede observar que todas las probetas entraron al medio de enfriamiento equitativamente.

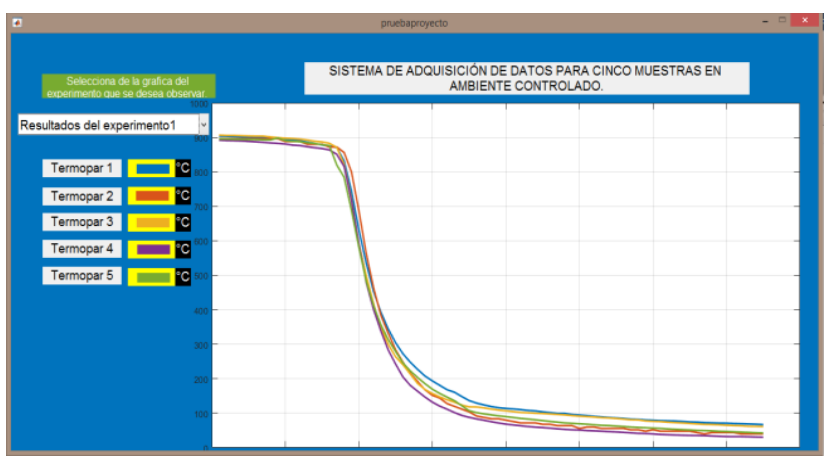

Gráfico 2 Comportamiento gráfico de los datos obtenidos del primer experimento

Fuente: elaboración propia

En el experimento dos, se observa en el Gráfico 3 dónde, la probeta 4 entro primero al medio de enfriamiento, para posteriormente ingresar las demás probetas, por tal motivo el comportamiento de la gráfica es de la cual se siguen obteniendo las seis lecturas por segundo.

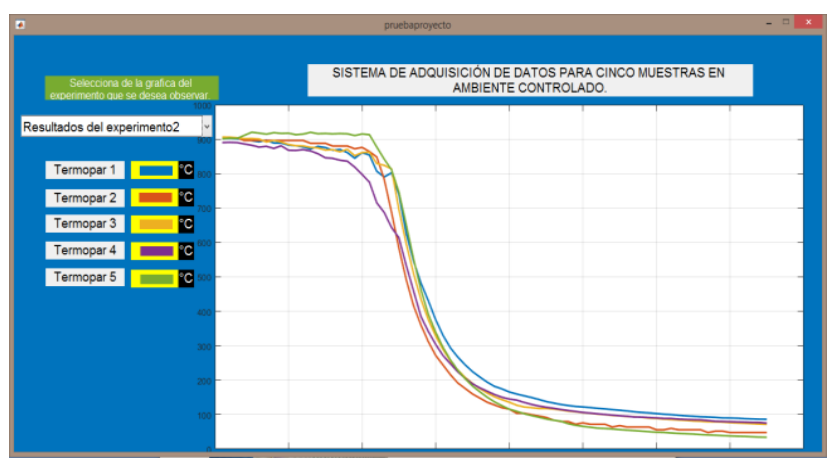

Gráfico 3 Comportamiento gráfico de los datos obtenidos del segundo experimento

Fuente: elaboración propia
En general, se experimentó que el comportamiento del equipo de adquisición de datos posee en todas las pruebas la característica de la obtención de seis lecturas por segundo. Para el experimento tres se realizó un análisis gráfico, del punto en el cual se somete al enfriamiento súbito de las probetas y se percibe que nuevamente la probeta 4 , es la que ingresa primero al medio de enfriamiento, seguido por las demás probetas Gráfico 4.

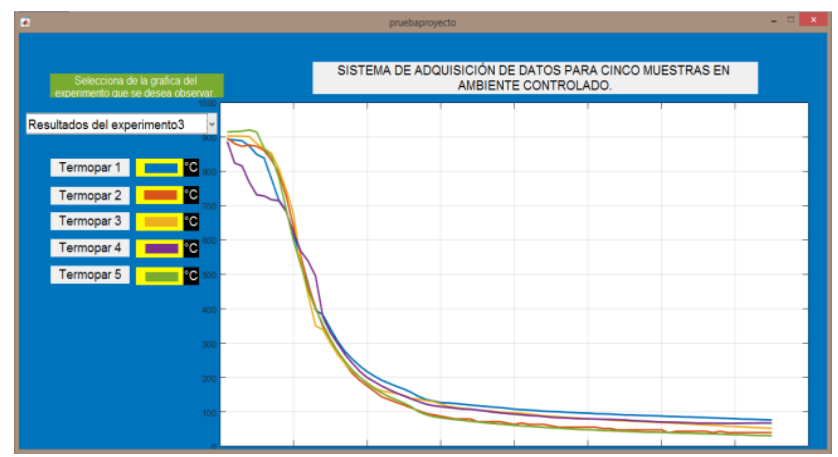

Gráfico 4 Comportamiento gráfico de los datos obtenidos del tercer experimento

Fuente: elaboración propia

El motivo por el cual se obtienen resultados muy distintos es por la forma en la cual se realizaron los demás experimentos, esto se debe principalmente a que cada uno se realizó de forma manual, lo que complica replicar el experimento varias veces.

Otro de los resultados obtenidos es la interfaz visual que tiene el equipo con la pantalla LCD 20X4 (Figura 5), lo fundamental de este elemento es que durante las pruebas se logró tomar decisiones en cuanto a fallas de lectura presentadas, ya sea a causa de un falso contacto con los termopares o problemas de lectura con los módulos de lectura MAX 6675.

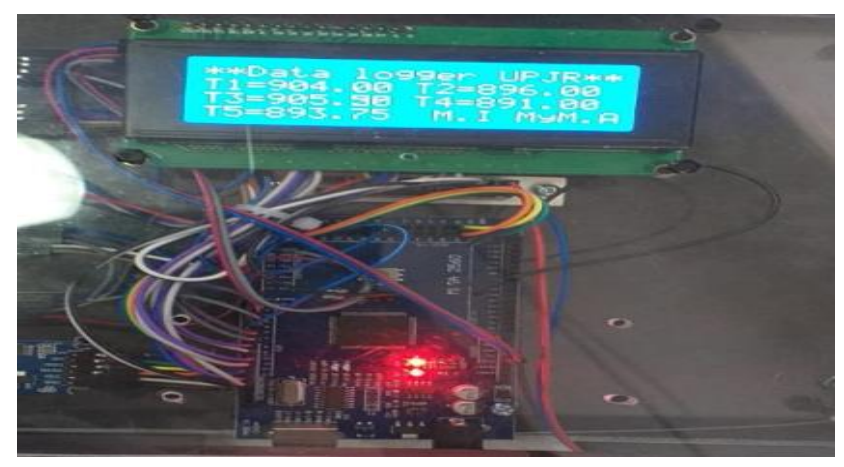

Figura 5 Sistema de recolección de datos en funcionamiento con la pantalla LCD Fuente: Imagen del Autor Ing. Benjamín Núñez Ariza 


\section{Agradecimiento}

Por brindarme la flexibilidad, el apoyo y las recomendaciones durante el desarrollo de este artículo, así como en el proceso de experimentación, al comité académico de la Maestría en Ingeniería de la Universidad Politécnica de Juventino Rosas.

\section{Conclusión}

El equipo cumple con las expectativas previstas, pues recolectó los datos esperados, se considera el mejoramiento del equipo, desde la parte del hardware hasta el software e incluso, el equipo de pruebas, el método de extracción del horno y el enfriamiento, debido a que este último proceso influye directamente en los resultados si se deseará hacer un estudio de tratamiento térmico.

Por tal razón, se le dará un seguimiento a este proyecto, con la finalidad de demostrar que este dispositivo es flexible, ergonómico y confiable, además que se puede adecuar a otros procesos industriales, por lo que se pretende adecuar para que pueda hacer lecturas de cualquier variable física de la que se busque generar datos. De igual manera la aplicación guide seguirá en desarrollo con el objetivo de mejorar la interacción con los gráficos.

\section{Referencias}

ASTM Designation: 255-2 Standard Test Methods for Determining Hardenability of Steel "THE SCIENCE AND ENGIEERING OF MATERIALS"

SEVENT EDITION, DONAL. ASKELAND AND WENDELING J. WRIGHT @2016,2011 Cengage Learning.

"LECTURAS
LABORATORIO DE

